\title{
An Emotion Model of 3D Virtual Characters In Intelligent Virtual Environment
}

\author{
Zhen Liu ${ }^{1}$, Zhi Geng Pan $^{2}$ \\ ${ }^{1}$ The Faculty of Information Science and Technology, Ningbo University, \\ 315211, China \\ liuzhen@nbu.edu.cn \\ ${ }^{2}$ State Key Lab of CAD\&CG, Zhejiang University, Hangzhou, 310027, China \\ zgpan@cad.zju.edu.cn
}

\begin{abstract}
Human emotion is related to stimulus and cognitive appraisal. Emotion is very important to entertainment application of virtual reality. Emotion model of 3D virtual characters is a challenging branch of Intelligent Virtual Environment (IVE). A believable 3D character should be provided with emotion and perception. In general, a virtual character is regarded as an autonomous agent with sense, perception, behavior and action. An emotion model of $3 \mathrm{D}$ virtual characters on the basis of psychology theory is presented in this paper. Our work is to construct 3D virtual characters that have internal sensor and perception for external stimulus, and express emotion autonomously in real time. Firstly, architecture of a virtual character is set up by cognitive model; Secondly, emotion class is set up by OCC and Plutchik's emotion theory; Thirdly, some new concepts about emotion are presented with a general mathematical model which is relation among emotion, stimulus, motivation variable, personality variable. Fourthly, a perception model of 3D characters by Gibson's theory is introduced. As a result, an emotional animation demo system of 3D virtual character is implemented on PC.
\end{abstract}

\section{Introduction}

Emotion is a complex subjective experience by outside stimulus. Emotion is very important to entertainment application of virtual reality. Emotion model of 3D virtual characters is a challenging branch of Intelligent Virtual Environment (IVE)[1]. A $3 \mathrm{D}$ virtual character is regarded as an autonomous agent and should be provided with the ability of emotion expression besides perception and behavior. Our research is mainly based on behavior animation [2][3][4], our goal is to construct 3D virtual characters with the ability of emotion self-control in IVE. The emotion model in this paper gives a quantitative description for emotion process.

The remainder of this paper is organized as follows: In the section 2, architecture of virtual character is described by cognitive model. In the section 3, emotion class of virtual characters is introduced. In the section 4 , an emotion model of virtual characters is presented, if the intensity of an emotion stimulus is bigger than the resistive intensity of an emotion, an emotion will occur. In the section 5, a new model of per- 
ception of 3D virtual character is presented. In the section6, emotional animation demo system of 3D virtual characters is realized. Finally, conclusion is in Section 7.

\section{Architecture of 3D Virtual Characters}

A virtual character can be regarded as an agent. An ideal agent is one that always does whatever action is expected to maximize its performance measure, on the basis of perception and built-in knowledge [3].

In this paper, architecture of virtual character is presented in Fig. 1.

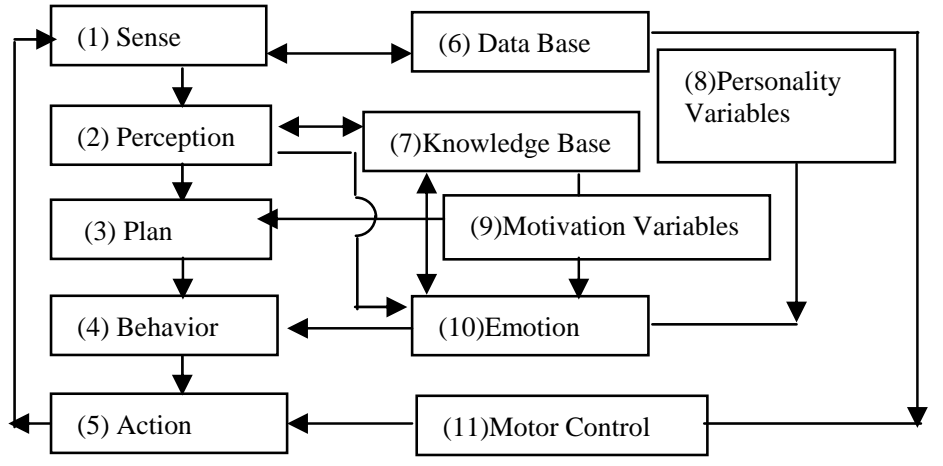

Fig. 1. Architecture of a 3D virtual character by cognitive model

(1) The sense module of character reads the database to the perception module.

(2) The perception module reads the knowledge base, and repairs the default motion plan and updates external stimulus.

(3) The plan module includes the current goals. It reads the knowledge base and makes behavior codes to behavior module.

(4) The behavior module is responsible for plan module and emotion module, and control the action module. Behaviors are expressed by a set of behavior codes, this module convert emotion expression code to behavior code.

(5) The action module uses the motion control module and executes the behavior. It will read motion capture data from database.

(6) The database module includes 3D geometry of virtual environment, original information and current state information, such as, the original location and parameters of virtual character, motion capture data, 3D model and location of objects, default motion plan scripts which record some goal location, and internal state for motivation variables.

(7) The knowledge base module includes some production rules in scripts that express the relations among motivation variable, emotion and behaviors.

(8) Personality variables are some stable psychological traits of a virtual character.

(9) Motivation variables include some physiology parameters of virtual character. For example, tiredness is one of motivation variables. We can set up a value between 0 and 1 to measure it. If the value is equal to 0 , the character is not tired, while if the 
value is equal to 1 , the character is too tired. These two different states will influence emotion variously.

(10) The emotion module receives external information from perception, and reads motivation and personality variables. If an emotion is active, this module will create emotion expression, emotion expression code will be sent to behavior module.

(11) The motion control module includes inverse kinematics arithmetic and motion captures arithmetic.

\section{Emotion Class of Virtual Characters}

There are some classical research works in emotion model. The theories of emotion in psychology [5] demonstrate that emotion is a cognitive interpretation of those responses to emotional experiences. Emotion associates the environment stimulus with the character personality on the basis of James-Lange theory of emotion and Schachter-Singer theory of emotion, and occurs with motivation simultaneously. In some sense, motivation can intensify emotion, but emotion can also create motivation. Emotion is usually transitory, with a relatively clear beginning and ending, and a short duration. Ortony et al set up an emotion cognitive model that is called OCC model [6]. In the model, emotions are generated in reaction to objects, actions of agents and events. They outlined specifications for 22 emotion types. Picard gave the concept of affective computing for interface between human and computer [7]. In the opinion of Plutchik's emotion classification [8], emotion intensity distributes on a "circle" with eight basic categories of emotion that motivate various kinds of adaptive behavior of character. In the center of "circle", emotion intensity is zero, while in the edge of "circle" emotion intensity is one. In this paper, the simplified Plutchik's emotion classification on face expression is as follows: happiness, surprise, sadness, fear, disgust, and anger.

This paper integrates OCC emotion model and Plutchik's emotion classification together; a virtual character can have 22 types emotion in OCC model, and six basic face expressions. We can set a function from OCC emotion types to six face expressions in table 1.

Table 1. Relation between Plutchik's simplified emotion types and OCC emotion types

\begin{tabular}{cl}
\hline Plutchik's types & Emotion types in OCC model \\
\hline Happiness & Happy-for, Gloating, Joy, Pride, Admiration, Love, \\
& Hope, Satisfaction, Relief, Gratification, Gratitude \\
Disgust & Hate \\
Anger & Anger, Reproach, Hate \\
Sadness & Resentment,Pity,Distress,Shame,Disappointment, \\
& Remorse \\
Fear & Fear, Fear-confirmed \\
Surprise & By context \\
\hline
\end{tabular}




\section{Emotion model of Virtual Characters}

In this section, we give some new definitions for describing emotion process of virtual characters.

Definition 1. For a certain virtual character, $\boldsymbol{B} \boldsymbol{E}$ is a basic emotion class set, $\boldsymbol{B} \boldsymbol{E}=\left\{\right.$ be $_{1}, \ldots$, be $\left.{ }_{\mathrm{N}}\right\}, \mathrm{i} \in[0, \mathrm{~N}]$, be ${ }_{i}$ is a basic emotion (such as happy-for). $\mathrm{N}$ is the number of basic emotion class.

Definition 2. For a certain virtual character, $t$ is time variable, $E(t)$ is emotion variable.

Definition 3. For an emotion variable $E(t), \Theta_{i}[E(t)]$ is membership function for emotion class be $_{i}, \Theta_{i}[E(t)] \in[0,1]$.

If $\Theta_{i}[E(t)]=0$, the virtual character has no emotion be ${ }_{i}$; If $\Theta_{i}[E(t)]=1$, the virtual character has emotion be ${ }_{i}$ with intensity $=1$. $^{2}$

Definition 4. For a certain virtual character, $O_{j}(t)$ is an external stimulus, $j \in[0, n o]$, no is the number of stimulus. $\Theta\left[O_{j i}(t)\right]$ is the stimulus intensity function of $O_{j}(t)$ for emotion class be ${ }_{i}, \Theta\left[O_{j i}(t)\right] \in[0,1]$.

Definition 5. For a certain external stimulus $O_{j}(t), \mathrm{d}_{j}(t)$ is distance from virtual character to center of stimulus $O_{j}(t)$.

In general, $\partial \Theta\left[O_{j i}(t)\right] / \partial \mathrm{d}_{j}(t)<0$, there exist two distance $\mathrm{D}_{\max }$ and $\mathrm{D}_{\min }$, if $\mathrm{d}_{j}(t)>$ $\mathrm{D}_{\max }, \Theta\left[O_{j i}(t)\right]=0$; if $\mathrm{d}_{j}(t)<\mathrm{D}_{\min }, \Theta\left[O_{j i}(t)\right]=1$.

Definition 6. For a certain virtual character, $C_{i}(t)$ is the resistive intensity of emotion class be ${ }_{i}, i \in[0, \mathrm{~N}]$, The weaker $C_{i}(t)$ of a virtual character is, the more emotional the virtual character becomes to be with emotion class be ${ }_{i} . C_{i}(t) \in[0,1]$.

Definition 7. For a certain virtual character, $P S_{k}(t)$ is the personality variable, $\Theta\left[P S_{k}(t)\right]$ is the intensity of $P S_{k}(t), k \in[1, n p s], n p s$ is the number of personality. $\Theta\left[P S_{k}(t)\right] \in[0,1]$. In this paper, we use the Five Factor Model Personality [9][10], and $\quad P S 1(t)=$ openness, $\quad P S_{2}(t)=$ conscientiousness, $\quad P S_{3}(t)=$ extraversion, $P S_{4}(t)=$ agreeableness, $P S_{5}(t)=$ neuroticism.

Definition 8. For a certain virtual character, $M V_{m}(t)$ is the motivation variable, $m$ $[1, w], w$ is the number of motivation variable. $\Theta\left[M V_{m}(t)\right]$ is the intensity of $M V_{m}(t)$, $\Theta\left[M V_{m}(t)\right] \in[0,1] . R_{m}$ is the weights of $M V_{m}(t), R_{m} \in[0,1]$, and

$$
\sum_{m=1}^{w} R_{m}=1 .
$$

In this paper, $w$ is 5 by Maslow's theory, and $M V_{1}(t)=$ Physiological, $M V_{2}(t)=$ Safety, $M V_{3}(t)=$ Affiliation, $M V_{4}(t)=$ Achievement, $M V_{5}(t)=$ Self-Actualization.

Definition 9. For a certain $C_{i}(t)$, personality has impact on emotion state, $\alpha_{k i}$ is an impact coefficient from personality $P S_{k}(t)$ to $C_{i}(t)$. $N C_{k i}(t)$ is the updating $C_{i}(t)$ with considering impact from personality $P S_{k}(t), N C_{k i}(t)=\min \left[\alpha_{k i} C_{i}(t), 1\right], \alpha_{k i}>=0$. If $\alpha_{k i}=1, N C_{k i}(t)=C_{i}(t)$, personality has no impact on resistive intensity of emotion. $N C_{i}(t)$ is the updating $C_{i}(t)$ with considering impact from all personality variable, 


$$
N C_{i}(t)=\sum_{k=1}^{n p s} \Theta\left[P S_{k}(t)\right] N C_{k i}(t) \quad / \sum_{k=1}^{n p s} \Theta\left[P S_{k}(t)\right] .
$$

Definition 10. For a certain $C_{i}(t)$, motivation has impact on emotion state, $\beta_{m i}$ is an impact coefficient from motivation variable $M V_{m}(t)$ to $C_{i}(t) . M C_{m i}(t)$ is the updating $C_{i}(t)$ with considering impact from motivation variable $M V_{m}(t), M C_{m i}(t)=\min \left[\beta_{m i}\right.$ $\left.C_{i}(t), 1\right], \quad \beta_{m i}>=0$. If $\beta_{m i}=1, M C_{m i}(t)=C_{i}(t)$, motivation variable has no impact on emotion. $M C_{i}(t)$ is the updating $C_{i}(t)$ with considering impact from all motivation variable, and so:

$$
M C_{i}(t)=\sum_{m=1}^{w} \Theta\left[M V_{m}(t)\right] R_{m} M C_{m i}(t) / \sum_{m=1}^{w} \Theta\left[M V_{m}(t)\right] R_{m} .
$$

Definition 11. For a certain $C_{i}(t)$, motivation and personality has impact on emotion state in the same time, $T C_{i}(t)$ is the updating $C_{i}(t)$ with considering impact both from personality and motivation. $T C_{i}(t)=\min \left(N C_{i}(t), M C_{i}(t)\right)$

Definition 12. For a certain external stimulus $O_{j}(t)$ and resistive intensity of emotion $T C_{i}(t)$, the emotion trigger of $O_{j}(t)$ can be expressed as:

If $\Theta\left[O_{j i}(t)\right]-T C_{i}(t)<0$, the emotion class be ${ }_{i}$ is not active, $\Theta_{j i}[E(t)]=0$; If $\Theta[O$ $\left.{ }_{j i}(t)\right]-T C_{i}(t)>0$, the emotion class be ${ }_{i}$ is active with the intensity $\Theta_{i}[E(t)]$, in this paper, $\Theta_{j i}[E(t)]=\left[\Theta\left[O_{j i}(t)\right]-T C_{i}(t)\right] /\left[1-T C_{i}(t)\right]$.

\section{Perception of 3D Virtual Characters}

3D Virtual characters should be equipped with visual, tactile and auditory sensors in order to execute the perception. This paper only discusses the visual perception of characters. Synthetic vision is an important method for visual perception [11], which can accurately simulate the vision from view of character. In this paper, each object is assigned a unique color code, to check which objects are visible to virtual character; the scene is rendered from the character's point of view with no texture (only with color by color ID for each object in Fig.2.), There are many characters in a complex IVE, synthetic vision will be costly. Furthermore, this method cannot get the semantic information for objects. Therefore, another method for simulation of visual perception is presented in this paper. Our method is based on the Gibson's theory of affordances[12], affordances are relations among space, time and action. A character can perceive these affordances directly. An affordance is invariance for environment. We set up some semantic information in database for special area or object in the 3D virtual environment. For example, when a character wants to walk across a road, only the zebra crossing is accessible, so that the character will select zebra crossing. Scene octree [11] can be used to simulate the character memory for static object in 3D virtual environment in our demo system. With doing so, some navigating points can be set up in a 3D environment, and a character can seek the navigating point that is near- 
est to him and move to it. Usually, a character moves from one navigating point to another. A default plan is a script file that records default-navigating points of a character. If there is no external stimulus, a character walks by a walking plan. When a character perceives an object or events, he may stop walking and make some actions (such as avoiding object), then he continues walking to the nearest navigating point.

The visual perception of virtual character is limited to a sphere, with a radius of $R$ and angle scope of $\theta$. We adapt the method of Tu et al [2]. The vision sensor is at point $O$ (the midpoint between the two eyes), and sets up local right-handed coordinate system. $O$ is the origin and $\boldsymbol{X}$ axis is along front orientation. To determine if the object $P$ is visible, the first step is to judge whether $P$ is in the vision scope. If $P$ is in the vision scope, the character detects whether other objects occlude it. The second step is to shoot a ray from $O$ to $P$. If $\|P-O\|<R$ and the angle between the ray and $X$ axis is less than $\theta / 2$, the object is visible. In a $3 \mathrm{D}$ virtual environment, there are a lot of dynamic objects, on which we set up feature points (such as geometric center). If one feature point is visible, the object is regarded as visible.

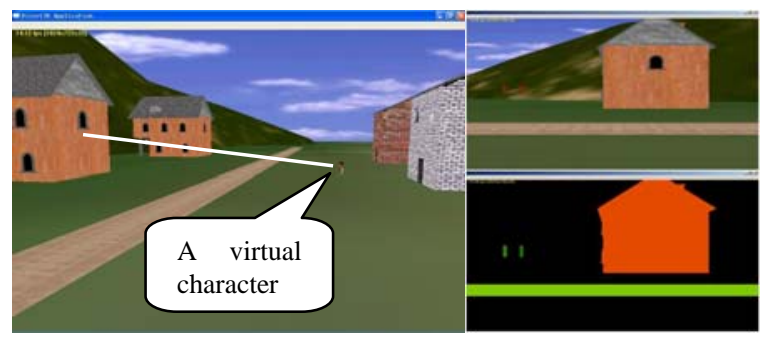

Fig. 2. A virtual character is watching a house on the left part of Fig.2; the right part of Fig. 2 is vision image and synthetic vision image (in the bottom right corner).

\section{Experimental Results}

A Demo system of 3D virtual characters animation is developed with Visual c++ and Direct3D. Unlike traditional computer animation in 3D animation tool (such as Maya), the character in the demo system is autonomous and behavioral, the virtual character have the ability to sense and perceive virtual environment, the emotion behavior was expressed by the body pose movement and facial expression, users can rove the virtual world and watch the virtual characters from any position. Emotion interactions between two characters are nonverbal, all information of nonverbal emotion interaction will be sent to blackboard (a block of common memory that is used to share communication information among characters), each character will perceive the emotion state of others. Body pose animation and facial expression animation are integrated together by analytical inverse kinematics method and motion captures data, geometry blending is used to create facial expression animation.

In order to simplify perception model of virtual character, attention mechanisms should be integrated in process of perception. Virtual characters need not focus to all 
objects in virtual environment by attention mechanism of psychology. An attention object list which virtual character could focus to can be set up beforehand for different virtual characters. If an object is in the scope of perception, but is not in attention object list, then the character will not perceive the object. A snapshot of our demo system is shown in Fig.3.

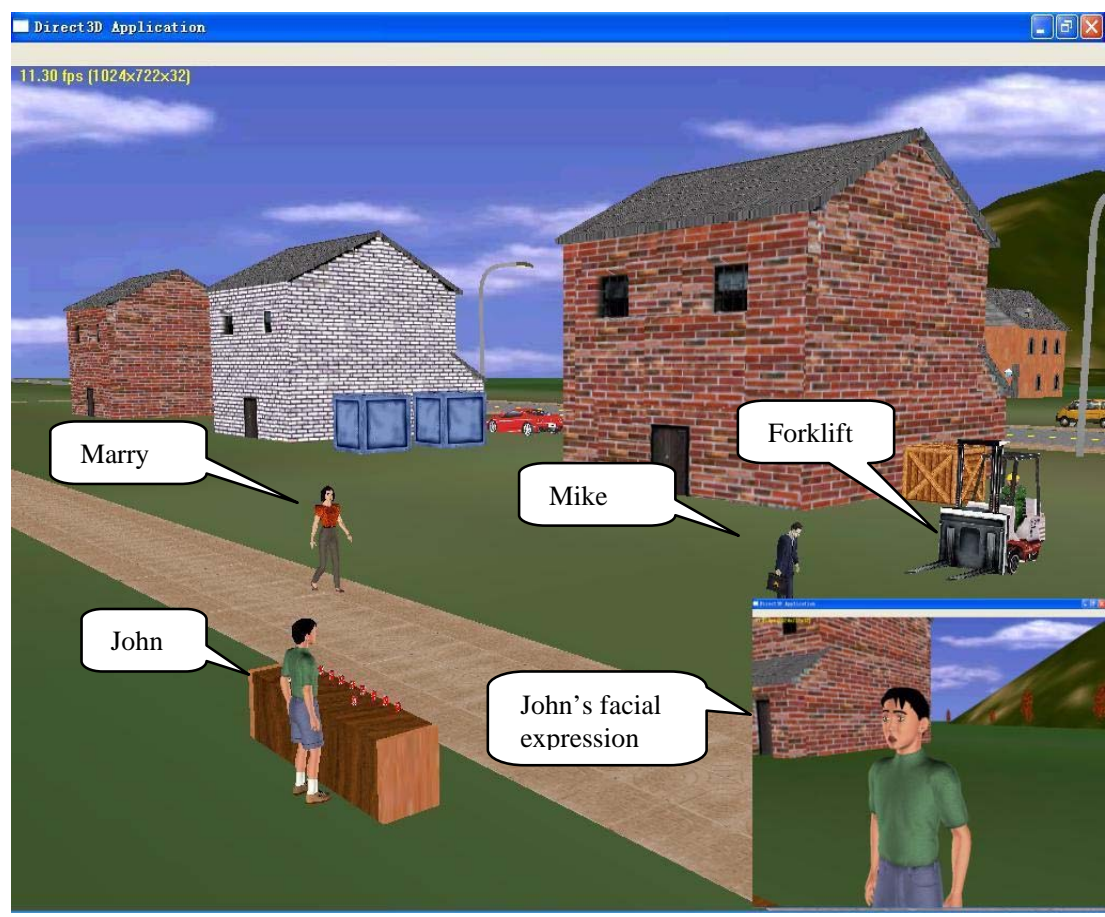

Fig. 3. John is selling drink, Marry is walking on road, Mike is searching on grass, a forklift is driving to Mike and John, when the forklift is near enough to John (the distance is less that $50 \mathrm{~m}$ ), John will express surprise emotion on face.

\section{Conclusion and future work}

The paper can be summarized as follows:

1.Architecture of 3D virtual characters is presented. This model uses the motivation variables and personality variables to express internal physiology and psychology parameters.

2. A visual perception model is presented, some semantic information is set up for special area in virtual environment, and scene octree is used to simulate the character memory for space.

3. Emotion is active from external stimulus. If an intensity of stimulus is bigger than the resistive intensity of emotion, that emotion will occur. A computational model of emotion is presented. 
4. A demo of emotional behavior animation is realized on PC.

The process of human emotion is very complex. this paper only gives a outline on stimulus and emotion. There is still much hard work to do. For the emotion model of virtual character in a large complex virtual environment, we hope to add uncertain reasoning models to describe emotion expression, and further study the emotion stimulus. Further more, we want to extend the demo system to construct emotional story and a virtual society of characters.

\section{References}

1. Aylett, R., Marc, C. : Intelligent Virtual Environments, A State-of-the art Report. Eurographics 2001, (2001) 87-109

2. Tu, X., Terzopoulos, D.: Artificial fishes: Physics, locomotion, perception, behavior, In Proc. SIGGRAPH'94 Conf, Orlando, FL USA, (1994) 43-50

3. Funge, J., Tu, X., Terzopoulos, D.: Cognitive Modeling: Knowledge, Reasoning and Planning for Intelligent Characters, In Proc. SIGGRAPH'99 Conf, Los Angeles, CA, (1999) 2938

4. Badler, N., Phillips, C., Webber, B.: Simulating Humans: Computer Graphics Animation and Control, New York: Oxford University Press (1993) 154-159

5. Bernstein, D.A., Stewart, A.C., Roy, E.J., Wickens, C.D.: Psychology (forth edition), New York: Houghton Miffin Company (1997) 360-361

6. Ortony, A., Clore, G.L., Collins, A.: The cognitive structure of emotions. New York: Cambridge University Press (1988)

7. Picard, R.W.: Affective Computing, MIT Press, London, England (1997)

8. Available online: http://www.wikipedia.org/wiki/Emotion

9. Egges, A., Kshirsagar, S., Thalmann, N.M.: Generic personality and emotion simulation for conversational agents, Computer Animation and Virtual Worlds, 15, (2004) 1-13

10. Gratch, J., Marsella, S.: A Domain-independent framework for modeling emotion, Journal of Cognitive Systems Research, 5(4), (2004) 269-306

11. Noser, H., Renault, O., Thalmann.D., Thalmann, N.M.: Navigation for Digital Actors Based on Synthetic Vision, Memory, and Learning, Computer \& Graphics, 19(1), (1995) 719

12. Gibson, J.J.: The ecological approach to visual perception.NJ:Lawrence Erlbaum Associates, Inc, Hillsdale(1986) 